\title{
Tissue segmentation: a crucial tool for quantitative MRI and visualization of anatomical structures
}

\author{
Fritz Schick $^{1}$
}

Published online: 6 April 2016

(C) ESMRMB 2016

\begin{abstract}
Automatic or semi-automatic segmentation of tissue types or organs is well established for X-ray-based computed tomography, with its fixed grey-scale and tissue classes with well-established ranges of Hounsfield units. MRI is much more powerful with regard to soft tissue contrast and quantitative assessment of tissue properties (e.g., perfusion, diffusion, fat content), but the principle of signal generation and recording in MRI leads to inherent problems if simple threshold based segmentation procedures are applied. In this editorial in the special issue of MAGMA on tissue segmentation, a number of relevant methodical, scientific, and clinical aspects of reliable tissue segmentation using data recording by MRI are reported and discussed.
\end{abstract}

Keywords MRI, tissue segmentation · MRI, tissue classes $\cdot$ MRI, image segmentation algorithms

The development of post-processing tools for medical images is an interdisciplinary research field at the intersection of medicine, physics, and computer science. Computational techniques for the automatic assessment of features of body parts from image data sets become increasingly important when high numbers of large data sets are recorded.

Tissue segmentation aims at partitioning an image into segments corresponding to different tissue classes. In healthy subjects, these classes are biologically defined as

Fritz Schick

fritz.schick@med.uni-tuebingen.de

1 Section On Experimental Radiology, Department of Diagnostic and Interventional Radiology, University of Tuebingen, Hoppe-Seyler-Str. 3, 72076 Tuebingen, Germany specific types of tissue, whole organs, or sub-regions of organs (e.g., liver or lung segments or muscle groups). In patients, areas with pathologies as tumours or inflammation can also be relevant for segmentation.

Clinical imaging, with its long tradition of radiography, works largely with the eyes and brains of well-trained radiologists. On the other hand, quantitative assessment of relevant clinical findings is important, as the description is thus reader-independent, and allows for reliable assessment of disease progression and treatment response in follow-up examinations. Thus, the long-standing practice has been that lesion dimensions are roughly measured using a ruler, and the number of visible lesions are counted manually.

Recent years have witnessed a paradigm change regarding the role of medical imaging in the clinic. Imaging is seen not only as a diagnostic tool for revealing diseases in a qualitative or semi-quantitative manner; rather, imaging techniques can and should be used for quantitative characterization of tissue volume or shape, chemical composition, and functional activity, if those data are helpful for answering clinical questions. Furthermore, studies are investigating imaging-related "biomarkers" that are useful for describing the activity of individual diseases [1-3].

The quality and reliability of the results of segmentation techniques depends not only on the computational algorithms applied, but also on the imaging modality, the setup, and the parameters chosen for data recording. Medical image segmentation usually works well in two-dimensional or three-dimensional data sets with high contrast between the tissue classes to be separated. High spatial resolution is helpful for avoiding partial volume effects. The noise level in the images should be low, and the grey values of the specific tissue classes in data sets should be independent of their spatial position. Furthermore, artificial signals which 
are not adequately correlated to structures in the body part examined should be absent.

Common imaging modalities useful for recording tomograms or isotropic three-dimensional data in human examinations include computed tomography (CT), magnetic resonance imaging (MRI), positron emission tomography (PET), and ultrasound (US). PET alone (without congruent CT or MR images) provides relatively low spatial resolution [4], and US images often show strong artefacts that may not be adequately handled by automatic segmentation software. Some structures, however, such as the faces of fetuses, can often be reconstructed well [5]. CT based on $\mathrm{X}$-ray absorption provides a favorable basis for automatic segmentation of several tissue classes, as grey values are given in well-defined Hounsfield units that directly reflect $\mathrm{X}$-ray absorption in the tissue [6]. Thus, picture elements in CT scans can be attributed to several tissue classes (e.g., soft tissue or bone) using relatively simple threshold approaches. Angiographic CT data sets (including coronary angiography) recorded after intravenous administration of contrast media can be successfully reconstructed using maximum-intensity projection techniques (MIPs) [7], but higher-quality and better visualization of the spatial relation between blood vessels and other body parts is achieved by surface reconstruction after tissue segmentation [8]. Related post-processing procedures are available on modern CT consoles working fully automatically. On the other hand, the recording of highly resolved data sets by CT is problematic because of inevitable radiation exposure (especially for multiple scanning sessions in functional studies), and soft tissue contrast of CT data is inherently inferior to that of MRI.

MRI is clearly the preferred imaging modality for most cohort studies with healthy volunteers and for studies requiring optimal soft tissue contrast. On the one hand, MRI, with its multiple forms of contrast weighting and its sensitivity to diffusion, perfusion, and chemical composition of tissues, is a highly attractive alternative. On the other hand, some characteristics of MR images create greater difficulty for automatic tissue segmentation than CT data sets. First, grey values in MR images are proportional to signal intensities recorded by the receiver coils rather than the signal intensity generated inside the tissue. Especially when using surface coils or coil arrays for recording sensitive data, one finds clearly variable grey values in the same image for a given type of tissue, depending on the distance of the tissue site from the receiver coils. A second factor (especially for higher field strength) is the occurrence of spatially dependent excitation flip angles, leading to non-uniform contrast weighting in MR images [9]. Furthermore, although different types of contrast weighting in MRI offer many opportunities for differentiating various types of tissue, several contrast weighting techniques must often be combined to achieve reliable differentiation of all desired tissue classes [10].

Distinguishing anatomically defined tissues areas with (nearly) equal composition and signal behaviour is an especially difficult task. Segmentation of different lung lobes or liver lobes or the identification of specific muscle groups surrounded by other muscles thus requires the inclusion of anatomical pre-knowledge in the segmentation algorithms [11].

Various methodical approaches have been developed and applied over the years for proper segmentation in MR images. It began with basic threshold- and clustering-based techniques, which were complemented by advanced algorithms incorporating more anatomical a priori information into the segmentation task. These developments have led to more adaptable and appropriate problem solution, and have enabled the analysis of complex image structures in two- and three-dimensional data sets. Automatic analysis of dynamic and real-time datasets has also become feasible [12].

1. The first medical image segmentation approaches were based largely on user-specified signal thresholds. Expansions of these manual threshold-based algorithms attempted to automatically determine the optimal threshold value by introducing additional information, e.g., the intra- and inter-class variance of grey values such as in Otsu's method [13], or by reformulating the segmentation task as a clustering problem with distance metrics ( $k$-means) or distribution models (expectation maximization). However, in MRI, all segmentation algorithms must deal with artefacts, intensity inhomogeneities, and possible low-contrast or noisy images, which can limit the capabilities of the algorithms applied.

2. To overcome these challenges and to increase accuracy and robustness, a number of techniques directly incorporate knowledge regarding the anatomical structure or other abstract a priori information such as in model-based approaches [14]. Clustering improves the smoothness of the segmentation result by taking the spatial neighborhood into account [15], thereby suppressing the effects of noise. In order to correct intensity inhomogeneities, the bias field of the image can be estimated before or even within the segmentation task.

3. In atlas-based segmentation, a few datasets are usually labelled by clinical experts to obtain a reference atlas dataset, which can be fitted directly or in a warped representation to the current unknown MR image by minimization of an energy function or other optimization procedures [16, 17]. The information in the reference atlases can be consolidated into a single atlas (statistical atlas) or can be used in a multi-atlas approach with 
majority voting. One drawback of atlas-based segmentation is the potential for inaccurate reference atlases, which might bias the solution. Other shape-based methods rely on templates that can be created from reference datasets. The template represents the starting point of procedures, leading to iterative adjustments to the new image in active-shape models or active-appearance models. The combination of various techniques results in hybrid methods that avoid the disadvantages of individual approaches.

4. In addition to fully automatic segmentation, some techniques enable interactive segmentation of tissues in the clinical routine based on a user-provided coarse initial segmentation or seed points. With active-contour models, the boundary of the initial course segmentation can be iteratively shifted to the real object boundary, resulting in refinement of the solution by minimizing error metrics. Seed points provide starting points for a region-growing or class-labelling algorithm for classification of unlabelled image points. A clear advantage here is that the segmentation process can be supervised by the operating expert. Thus a reliable solution is guaranteed, often with an implementation-dependent possibility to immediately correct the segmentation results $[18,19]$.

The effective application of tissue segmentation in MRI is steadily growing. Quantitative assessment of tissue crosssectional data from two-dimensional MRI and volumes of anatomical structures in isotropic three-dimensional data sets have been shown to be helpful in many areas of medical research, and results can be used as biomarkers for diseases. For example, metabolic studies have shown high correlations between the individual amount of visceral fat and insulin resistance [20]. Examinations in Alzheimer patients have revealed that volumetric analysis of the hippocampus provides a reliable imaging-based biomarker for characterizing the disease [21]. A further application of tissue segmentation is the automatic assessment of interesting tissue properties as perfusion or diffusion. For this task, data with different (anatomical and functional) contrasts must be recorded and registered, especially when body regions with physiological motion (breathing or cardiac action) are involved [22]. Improved visualization of angiograms or the (semi)automatic separation of single arteries or differentiation of arterial and venous vessels can be supported by adapted segmentation strategies [23].

This topical issue on tissue segmentation covers review papers on both technical aspects of automatic and semiautomatic tissue segmentation and major current fields of application for different organ systems, written by experts in their fields. Seven original papers were selected that provide insight into interesting current research areas.
The work by Wang et al. [24] provides an up-to-date overview of the principles and methods commonly applied to achieve superior tissue segmentation results in MRI. The authors report on the impacts of MR image acquisition on segmentation outcome and the principles of selecting and exploiting segmentation techniques. Exemplary applications are visualized for better understanding.

The review article by Helms [25] reports on concepts of voxel-based and surface-based segmentation of different regions of the brain. Special emphasis is given to the typical contrast features and morphological constraints of cortical and subcortical grey matter. The paper by De Leener et al. [26] reviews the existing methods for spinal cord segmentation, including intensity-based, surface-based, and image-based approaches. Recommendations for validating spinal cord segmentation techniques are included, as this is considered to be especially important in this field.

Cardiac segmentation for computer-aided functional analysis of cardiac disease has become popular over the past two decades. The review article written by Peng et al. [27] illustrates and discusses automatic and semiautomatic approaches for delineation of the heart in four-dimensional MRI data. The original article contributed by Rusinek et al. [28] reports on applications of a semi-automatic procedure for the assessment of kidney volumes in ten diabetic and ten healthy subjects.

The review article contributed by Pedoia et al. [29] presents some of the techniques proposed in the recent literature that have been adopted in clinical studies for joint and musculoskeletal tissue analysis, in arthritis patients in particular. The authors discuss the MRI sequences and image processing algorithms most commonly used to address segmentation challenges. Three original papers on musculoskeletal applications are included in this special issue as well. The work of Sdika et al. [30] is dedicated to automatic assessment of individual muscles in the rat leg using a multi-atlas segmentation method. Karampatos et al. [31] tested the software package "sliceOmatic" in 21 human subjects to assess the reliability of its application in the lower leg. A study by Le Troter et al. [32] reports on automated segmentation of human thigh muscles and subsequent quantification of fatty infiltration in these muscles.

Assessment of locations and volumetric assessment of white and brown adipose tissue has become very important in recent years for metabolically oriented animal and human studies. The review article by $\mathrm{Hu}$ et al. [33] on segmentation techniques for adipose tissue gives the reader a solid basis of understanding regarding recent segmentation activities in this field of research. The authors discuss image post-processing techniques and emerging atlasbased automated segmentation techniques. The first original paper, by Bhanu Prakash et al. [34], reports on automated segmentation of brown fat in mice at 9.4 Tesla based 
on image data recorded with multi-point Dixon fat-water separation. The second paper [35], from the same group, is aimed at the reliable assessment of several different tissue types in rats using multi-parametric MRI with assessment of fat fraction, $\mathrm{T} 2$, and $\mathrm{T} 2 *$ values.

Last but not least is the paper from Ruhlmann et al. [36], which deals with proper tissue segmentation based on MRI data for PET attenuation correction in clinical oncologic MR-PET examinations. The authors reveal the negative effects of gadolinium contrast media on tissue segmentation based on Dixon imaging.

The interdisciplinary field of tissue segmentation might be seen as somewhat "exotic" by many "traditional" MR physicists and clinicians. However, adaption of segmentation techniques to the specific peculiarities of MRI and the adaptation of MR signal recording to the demands of segmentation algorithms is a truly challenging research field. So let us venture into this new field and contribute through studies and related work.

Acknowledgments Several people provided strong support for this special issue of MAGMA: Marguerite Izquierdo from the editorial office was always a very professional partner throughout the preparation of this issue, and she always provided most helpful advice. Andreas Zimmermann supported the selection of and correspondence with contributing scientists, and Martin Schwartz supported this issue with his expertise on segmentation strategies and algorithms.

\section{References}

1. Taylor AJ, Salerno M, Dharmakumar R, Jerosch-Herold M (2016) T1 mapping: basic techniques and clinical applications. JACC Cardiovasc Imaging 9:67-81

2. He N, Ling H, Ding B, Huang J, Zhang Y, Zhang Z, Liu C, Chen K, Yan F (2015) Region-specific disturbed iron distribution in early idiopathic Parkinson's disease measured by quantitative susceptibility mapping. Hum Brain Mapp 36:4407-4420

3. Machann J, Bachmann OP, Brechtel K, Dahl DB, Wietek B, Klumpp B, Häring HU, Claussen CD, Jacob S, Schick F (2003) Lipid content in the musculature of the lower leg assessed by fat selective MRI: intra- and interindividual differences and correlation with anthropometric and metabolic data. J Magn Reson Imaging 17:350-357

4. Laforest R, Liu X (2008) Image quality with non-standard nuclides in PET. Q J Nucl Med Mol Imaging 52:151-158

5. Kurjak A, Azumendi G, Andonotopo W, Salihagic-Kadic A (2007) Three- and four-dimensional ultrasonography for the structural and functional evaluation of the fetal face. Am J Obstet Gynecol 196:16-28

6. Bydder GM, Kreel L (1979) The temperature dependence of computed tomography attenuation values. J Comput Assist Tomogr 3:506-510

7. Prokop M, Shin HO, Schanz A, Schaefer-Prokop CM (1997) Use of maximum intensity projections in $\mathrm{CT}$ angiography: a basic review. Radiographics 17:433-451

8. Fishman EK, Ney DR, Heath DG, Corl FM, Horton KM, Johnson PT (2006) Volume rendering versus maximum intensity projection in CT angiography: what works best, when, and why. Radiographics 26:905-922
9. Hoult DI (2000) Sensitivity and power deposition in a high-field imaging experiment. J Magn Reson Imaging 12:46-67

10. Will S, Martirosian P, Würslin C, Schick F (2014) Automated segmentation and volumetric analysis of renal cortex, medulla, and pelvis based on non-contrast-enhanced T1- and T2-weighted MR images. Magn Reson Mater Phy 27:445-454

11. Karlsson A, Rosander J, Romu T, Tallberg J, Grönqvist A, Borga M, Dahlqvist Leinhard O (2015) Automatic and quantitative assessment of regional muscle volume by multi-atlas segmentation using whole-body water-fat MRI. J Magn Reson Imaging 41:1558-1569

12. Queirós S, Barbosa D, Heyde B, Morais P, Vilaça JL, Friboulet D, Bernard O, D'hooge J (2014) Fast automatic myocardial segmentation in 4D cine CMR datasets. Med Image Anal 18:1115-1131

13. Otsu N (1979) A threshold selection method from gray-level histograms. IEEE Trans Syst Man Cybern 9:62-66

14. Collins DL, Holmes CJ, Peters TM, Evans AC (1995) Automatic 3-D model-based neuroanatomical segmentation. Hum Brain Mapp 3:190-208

15. Ahmed MN, Yamany SM, Mohamed N, Farag AA, Moriarty T (2002) A modified fuzzy C-means algorithm for bias field estimation and segmentation of MRI data. IEEE Trans Med Imaging 21:193-199

16. Heckemann RA, Hajnal JV, Aljabar P, Rueckert D, Hammers A (2006) Automatic anatomical brain MRI segmentation combining label propagation and decision fusion. Neuroimage $33: 115-126$

17. Bai W, Shi W, Ledig C, Rueckert D (2015) Multi-atlas segmentation with augmented features for cardiac MR images. Med Image Anal 19:98-109

18. Yushkevich PA, Piven J, Hazlett HC, Smith RG, Ho S, Gee JC, Gerig G (2006) User-guided 3D active contour segmentation of anatomical structures: significantly improved efficiency and reliability. Neuroimage 31:1116-1128

19. Gering DT, Nabavi A, Kikinis R, Hata N, O’Donnell LJ, Grimson WEL, Jolesz FA, Black PM, Wells WM III (2001) An integrated visualization system for surgical planning and guidance using image fusion and an open MR. J Magn Reson Imaging 13:967-975

20. Miyazaki Y, Glass L, Triplitt C, Wajcberg E, Mandarino LJ, DeFronzo RA (2002) Abdominal fat distribution and peripheral and hepatic insulin resistance in type 2 diabetes mellitus. Am J Physiol Endocrinol Metab 283:E1135-E1143

21. Teipel SJ, Grothe M, Lista S, Toschi N, Garaci FG, Hampel $\mathrm{H}$ (2013) Relevance of magnetic resonance imaging for early detection and diagnosis of Alzheimer disease. Med Clin North Am 97:399-424

22. Ceritoglu C, Oishi K, Li X, Chou MC, Younes L, Albert M, Lyketsos C, van Zijl PC, Miller MI, Mori S (2009) Multi-contrast large deformation diffeomorphic metric mapping for diffusion tensor imaging. Neuroimage 15(47):618-627

23. van't Klooster R, de Koning PJ, Dehnavi RA, Tamsma JT, de Roos A, Reiber JH, van der Geest RJ (2012) Automatic lumen and outer wall segmentation of the carotid artery using deformable three-dimensional models in MR angiography and vessel wall images. J Magn Reson Imaging 35:156-165

24. Wang L, Chitiboi T, Meine H, Günther M, Hahn HK (2016) Principles and methods for automatic and semi-automatic tissue segmentation in MRI data. Magn Reson Mater Phy 29:95-110

25. Helms G (2016) Segmentation of human brain using structural MRI. Magn Reson Mater Phy 29:111-124

26. De Leener B, Taso M, Cohen-Adad J, Callot V (2016) Segmentation of the human spinal cord. Magn Reson Mater Phy 29:125-153

27. Peng P, Gooya A, Lekadir K, Shao L, Frangi A (2016) A review of recent advances in automatic MRI cardiac segmentation methods. Magn Reson Mater Phy 29:155-195 
28. Rusinek H, Lim JC, Wake N, Seah J, Botterill E, Farquharson S, Lim RP (2016) Blanket method for renal segmentation. Magn Reson Mater Phy 29:197-206

29. Pedoia V, Majumdar S, Link T (2016) Review: segmentation of joint and musculoskeletal tissue in the study of arthritis. Magn Reson Mater Phy 29:207-221

30. Sdika M, Tonson A, Le Fur Y, Cozzone PC, Bendahan D (2016) Multi-atlas-based fully automatic segmentation of individual muscles in rat leg. Magn Reson Mater Phy 29:223-235

31. Karampatos S, Papaioannou A, Beattie KA, Maly MR, Chan A, Adachi JD, Pritchard JM (2016) The reliability of a segmentation methodology for assessing intramuscular adipose tissue and other soft-tissue compartments of lower leg MRI images. Magn Reson Mater Phy 29:237-244

32. Le Troter A, Fouré A, Guye M, Confort-Gouny S, Mattei JP, Gondin J, Salort-Campana E, Bendahan D (2016) Volume measurements of individual muscles in human quadriceps femoris using atlas-based segmentation approaches. Magn Reson Mater Phy 29:245-257
33. Hu HH, Chen J, Shen W (2016) Segmentation and quantification of adipose tissue by magnetic resonance imaging. Magn Reson Mater Phy 29:259-276

34. Bhanu Prakash KN, Srour H, Velan SS, Chuang KH (2016) A method for the automatic segmentation of brown adipose tissue. Magn Reson Mater Phy 29:287-299

35. Bhanu Prakash KN, Verma SK, Yaligar J, Goggi J, Gopalan V, Lee SS, Tian X, Sugii S, Khee Shing Leow M, Bhakoo K, Velan SS (2016) Segmentation and characterization of interscapular brown adipose tissue in rats by multi-parametric magnetic resonance imaging. Magn Reson Mater Phy 29:277-286

36. Ruhlmann V, Heusch P, Kühl H, Beiderwellen K, Antoch G, Forsting M, Bockisch A, Buchbender C, Quick HH (2016) Potential influence of Gadolinium contrast on image segmentation in MR-based attenuation correction with Dixon sequences in whole-body 18F-FDG PET/MR. Magn Reson Mater Phy 29:301-308 